\title{
Application of the Conditional Nonlinear Optimal Perturbations Method in the Shallow Lake Ecological Degradation and Restoration
}

\author{
Bo Wang ${ }^{1,2,3}$ and Qianqian $\mathbf{Q i}^{3}$ \\ ${ }^{1}$ Institute of Applied Mathematics, Henan University, Kaifeng, Henan 475004, China \\ ${ }^{2}$ LASG, Institute of Atmospheric Physics, Chinese Academy of Sciences, Beijing 100029, China \\ ${ }^{3}$ School of Mathematics and Statistics, Henan University, Kaifeng, Henan 475004, China \\ Correspondence should be addressed to Bo Wang; wangbo_sdu@163.com
}

Received 19 June 2014; Accepted 2 September 2014

Academic Editor: Hann-Ming H. Juang

Copyright (C) 2015 B. Wang and Q. Qi. This is an open access article distributed under the Creative Commons Attribution License, which permits unrestricted use, distribution, and reproduction in any medium, provided the original work is properly cited.

\begin{abstract}
In the shallow lake ecosystems, the recovery of the aquatic macrophytes and the increase in the water transparency have been the main contents of the ecological restoration. Using the shallow lake ecological degradation and restoration model, CNOP method is adopted to discuss the instability and sensitivity of the ecosystem to the finite-amplitude perturbations related to the initial condition and the parameter condition. Results show that the linearly stable clear (turbid) water states can be nonlinearly unstable with the finite-amplitude perturbations, which represent the nature factors and the human activities such as the excessive harvest of the macrophytes and the sediment resuspension caused by artificially dynamic actions on the ecosystems. The results also support the viewpoint of Scheffer et al., whose emphasis is that the facilitation interactions between the submerged macrophytes and the water transparency are the main trigger for an occasional shift from a turbid to a clear state. Also, by the comparison with CNOPI, CNOP-P, CNOP, and (CNOP-I, CNOP-P), results demonstrate that CNOP, which is not a simple combination of CNOP-I and CNOP-P, could induce the shallow lake ecosystem larger departure from the same ground state rather than CNOP-I, CNOP-P, and (CNOP-I, CNOP-P).
\end{abstract}

\section{Introduction}

The shallow lakes, as one kind of wetlands, have been the important surface water resources. Now, they are suffering from the unprecedented pressure and will be involved into the degraded turbid water states with the excessive input of the nutrient loading, the rising water level frequently, the macrophytes deterioration for the storm, and so forth. The submersed macrophytes can reduce the sediment resuspension, absorb the nutrients from the water or the sediment, and decrease the nutrient loading. So, the recovery of the aquatic macrophytes and the increase in the water transparency have been the main contents of the ecological restoration in the shallow lake ecosystems. The positive feedback in natural systems is very common. The facilitation interaction is one of the positive feedback loops and can cause the turbid and the clear state to be alternative attractors. There exist facilitation interactions between submerged macrophytes and water transparency in the lake ecosystems $[1,2]$ : the growth of the submerged macrophytes will increase the water transparency, while the water transparency will promote the growth of the submerged macrophytes.

Over the last decades, in the research of stability and the regime shift about the shallow lake ecosystem, Beisner et al. thought that there exist two ways for the ecosystem to have a transition [3]; Scheffer et al. pointed out that the shallow lakes can exist in two alternative stable states: one is a clear water state dominated by rooted macrophytes with a diverse biological community; the other is a turbid state, dominated by high phytoplankton populations with few macrophytes and low biological diversity [4]. Also, Scheffer and Carpenter concluded that although field observation can provide hints of alternative stable states, experiments and models are essential for a good diagnosis [5]. Considering 


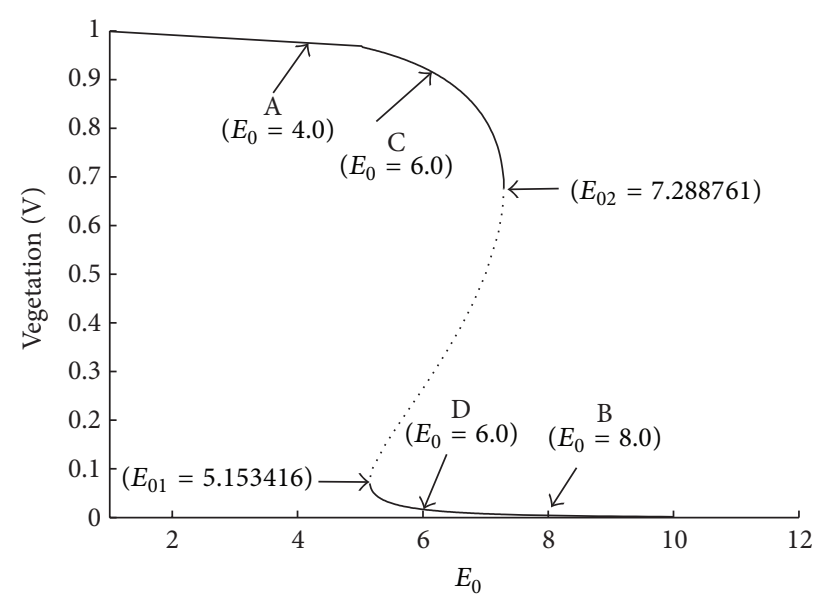

(a)

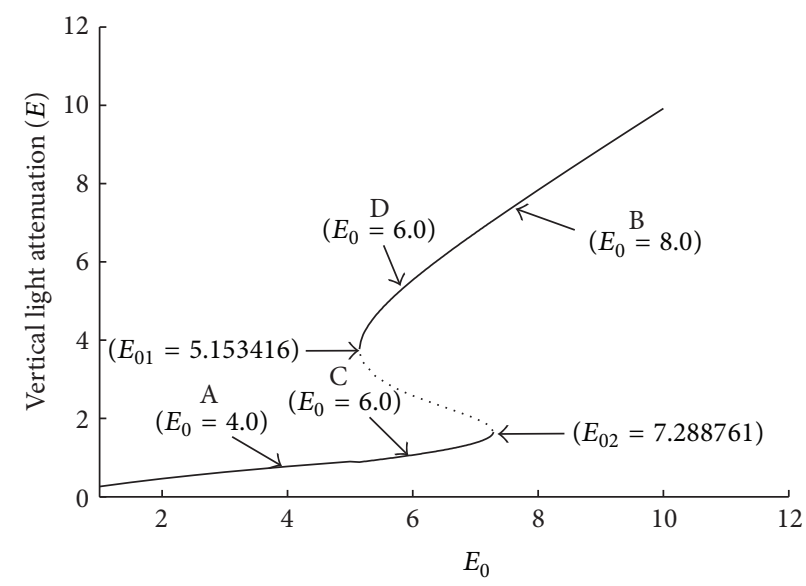

(b)

FIGURE 1: The equilibrium states versus the control parameter $E_{0}$; solid and dashed lines refer to linearly stable and unstable equilibrium states, respectively. (a) The equilibrium states are macrophyte covers. (b) The equilibrium states are the vertical light attenuation.

the potentially important complexity of a real ecosystem, Van Nes and Scheffer systematically explored the effect of spatial heterogeneity on regime shift for three different models [6]. By analyzing the model of Scheffer et al., Van Geest et al. found that shifts between the two contrasting states are common and episodes of the low water levels are an important external driver [7]. Here, to study whether and how the transitions between the different equilibrium states occur, we still consider the two-variable shallow lake ecosystem model [8], which is established by Scheffer. Using the model, on the one hand, we prove that there exist two equilibrium states: one is stable clear equilibrium states (CES) and the other is stable turbid equilibrium states (TES). On the other hand, we investigate how the initial perturbation and parameter perturbation in the given constraints affect the shallow lakes to the greatest extent.

In this paper, we use CNOP method to find the initial perturbation and model parameter perturbation of the largest evolution in given constraints at the prediction time. The method is proposed and extended by $\mathrm{Mu}$ et al. $[9,10]$ and the corresponding algorithm has been studied by Sun et al. [11]. The method has been used to study the problems of ENSO by Duan et al. [12-20] and the problems of ecology by Wang et al. [21-23]. In our previous work, we have studied the problem of the lake eutrophication using the single-variable model with CNOP method, which can be referred to in this journal in 2012. Here, CNOP method is adopted to study the problems of the shallow lake ecological degradation and restoration further. By comparing the impacts on the lakes with the different optimal perturbations, we can take proper measures to avoid the degradation of the ecosystem.

In the next section, the model and the approach of the CNOP will be described. Section 3 is concerned with the instability and the sensitivity of the shallow lake ecosystem by using CNOP-I, CNOP-P, and CNOP method. In Section 4, we will make a comparison on the effect on the shallow lake ecosystem with different optimal perturbations CNOP-I, CNOP-P, and CNOP and the simple combination (CNOP-I, CNOP-P). Finally, the results obtained in this paper are summarized in Section 5.

\section{The Model and the Method}

2.1. The Model. We consider the two-variable model of Scheffer [8], which describes the relation between macrophytes covers $V$ and turbidity $E$ in shallow lakes. The model is as follows:

$$
\begin{aligned}
& \frac{\mathrm{d} E}{\mathrm{~d} t}=r_{E} E\left(1.0-\frac{E}{E_{0}} \frac{h_{v}+V}{h_{v}}\right), \\
& \frac{\mathrm{d} V}{\mathrm{~d} t}=r_{V} V\left(1.0-V \frac{h_{E}^{p}+E^{p}}{h_{E}^{p}}\right),
\end{aligned}
$$

where the dynamical variable $V$ is the macrophyte covers and $E$ means the vertical light attenuation in the shallow lakes, which characterises the turbidity in water. $E_{0}$ is the control parameter, which represents the vertical light attenuation without macrophytes and $E_{0}$ can be related to the algal growth and resuspension of sediments in the shallow lakes. Based on [7], the turbidity of the lake without macrophytes is assumed to be dependent on the nutrient status. The rate parameters $r_{V}$ and $r_{E}$ determine how fast macrophytes and turbidity approach their equilibrium, and the values are 0.05 and 0.1 respectively. The power $p$ is $4.0 . h_{V}$ determines the macrophyte cover where the light attenuation is reduced by $50 \%$ and the value is 0.2 . $h_{E}$ represents the critical light attenuation around which the lake becomes vegetated and its value is 2.0. All the detailed descriptions of the model are consistent with those in the study by Van Geest et al. [7].

In this paper, we need two bifurcation diagrams about the macrophytes covers $V$ and the turbidity $E$ related to the control parameter $E_{0}$, respectively, and the exact values of the bifurcation points, which are not given by Scheffer et al. Here, using the Lyapunov stability theory and the fourth-order Runge-Kutta method, we get the two bifurcation diagrams which are plotted in Figure 1 and find the bifurcation points at $E_{01}$ and $E_{02}$ where $E_{01}=5.153418$ and $E_{02}=7.288761$. 
2.2. The Conditional Nonlinear Optimal Perturbations. Now, let us give a brief introduction to the method of CNOP. We write the evolution equations for the state vector $U$ and assume that the model is as follows:

$$
\frac{\partial U}{\partial t}=F(U, P),\left.\quad U\right|_{t=0}=U_{0}, \quad \text { in } \Omega \times[0, \tau],
$$

where $U(x, t)=\left(U_{1}(x, t), U_{2}(x, t), \ldots, U_{n}(x, t)\right)$ and $U_{0}$ is its initial states; $\Omega$ is a domain in $R^{n}, x=\left(x_{1}, x_{2}, \ldots, x_{n}\right), t=0$, is the initial time, and $t=\tau,(x, t) \in \Omega \times[0, \tau]$, with $\tau<+\infty$, is a future time; and $P$ represents the model parameter which is independent of time $t . F$ is a nonlinear differential operator. Assume that dynamical system equation (2) and the initial state are known exactly; the future state can be determined by integrating (2). The solution to (2) for the state vector $U$ at time $\tau$ is given by

$$
U(\tau)=M_{\tau}(P)\left(U_{0}\right) .
$$

Here, $M_{\tau}(P)$ is the propagator of (2) with the parameter vector $P$ from the time 0 to the time $\tau$ in the future.

Now we explore the situation that there exist both initial perturbation and parameter perturbation in (3). Then we have

$$
U(\tau)+u\left(u_{0}, p^{\prime} ; \tau\right)=M\left(P+p^{\prime}\right)\left(U_{0}+u_{0}\right)
$$

where $u\left(u_{0}, p^{\prime} ; \tau\right)$ is the departure from the reference state $U(\tau)$ caused by the combined error model $\left(u_{0}, p^{\prime}\right)$.

A nonlinear optimization problem is defined as follows:

$$
\begin{gathered}
J\left(u_{0} ; p^{\prime}\right)=\left\|M_{\tau}\left(P+p^{\prime}\right)\left(U_{0}+u_{0}\right)-M_{\tau}(P)\left(U_{0}\right)\right\|, \\
J\left(u_{0 \delta} ; p_{\sigma}^{\prime}\right)=\max _{u_{0} \in C_{\delta}, p^{\prime} \in C_{\sigma}} J\left(u_{0} ; p^{\prime}\right) .
\end{gathered}
$$

Here, $u_{0} \in C_{\delta}$ and $p^{\prime} \in C_{\sigma}$, respectively, are the constraint conditions of the initial perturbations and parameter perturbations. So, CNOP is the optimal combined mode of initial perturbations and parameter perturbations, which, for the given constraint, induces the largest departure from the reference state at time $\tau$. Particularly, only considering the initial perturbation, namely, $p^{\prime}=0$, the initial perturbation $u_{0 \delta}$ which satisfies the optimization problem is CNOP-I; when only investigating the effect of the model parameter perturbations on a given reference state, namely, we neglect the initial perturbation and take the constraints of initial perturbation as $u_{0}=0$, the parameter perturbation $p_{\sigma}^{\prime}$ which satisfies the optimization problem is CNOP-P.

Here, we use the fourth-order Runge-Kutta method to discrete the equation with $\mathrm{d} t=0.1$, which represents 0.1 years. It is obvious that the gradient is important for capturing the maximal value of the objective function. The gradient of the objective function with respect to initial perturbations and parameter perturbations is obtained by the adjoint method. With the spectral projected gradient (SPG) method, the CNOP-Is, CNOP-Ps, and CNOPs are obtained numerically.

\section{The Nonlinear Instability and the Sensitivity Analysis}

For different values of the parameter $E_{0}$, the model has different equilibrium states. Figures 1(a) and 1(b) show that the parameters $E_{01}$ and $E_{02}$ at the bifurcation points separate the interval into three parts: $E_{0}<E_{01}, E_{01}<E_{0}<$ $E_{02}$, and $E_{0}>E_{02}$. For $E_{0}<E_{01}$, there exists only one linearly stable CES (solid line), under which the water is clear and the macrophyte cover is high. When $E_{0}>E_{02}$, there just exists one linearly stable TES (solid line), under which the macrophyte cover is low and there exists too much phytoplankton in lakes. When $E_{01}<E_{0}<E_{02}$, there are one linearly unstable state (dashed line) and two linearly stable states (one is CES and the others are TES, solid line).

3.1. The Impact of CNOP-Is on the Shallow Lake Ecosystem. Using the spectral projected gradient method with the optimal time $\tau=100$, that is, 10 years, we obtain CNOP-Is under different control parameters $E_{0}$ and different initial constraint conditions. Here, the dynamical variables $V$ and $E$ should be nonnegative and so the initial perturbation $\left(V^{\prime}, E^{\prime}\right)$ should satisfy both the relation $V+V^{\prime} \geq 0, E+E^{\prime} \geq 0$ and the restraint condition $\left\|\left(V^{\prime}, E^{\prime}\right)\right\| \leq \delta$, where the 2-norm is employed to measure the initial perturbation. In the real ecosystem, the initial perturbations which relate to the human activities may represent the excessive harvest of the macrophytes, the use of the herbicides in lakes, wave breaking, turbulence prevention, and so on. All these factors will affect the macrophyte covers and the turbidity in water directly at the initial time.

The impact of CNOP-I on the ecosystem has been discussed in the early paper [24]. For simplicity, we just state the conclusion. When the control parameters $E_{0}<E_{01}$ and $E_{0}>E_{02}$, the numerical experiments show that, no matter how large the $\delta$ is, the ecosystem will return to the corresponding CES or TES, respectively, finally. The larger $\delta$ is, the longer time will be used for the ecosystem to return to CES or TES. When the control parameter $E_{0}$ is in the interval $\left[E_{01}, E_{02}\right]$, there exists the critical constraint value $\delta_{c}$. If $\delta<$ $\delta_{c}$, CNOP-Is induced the shallow lakes to return to its original TES or CES through its inner facilitation interactions and will not yield the transition. If $\delta>\delta_{c}$, the shallow lakes will transfer from one equilibrium state to another equilibrium state with corresponding CNOP-Is. In addition, we get the critical restraint value $\delta_{c}$ for the control parameter $E_{0}$ in the interval $\left[E_{01}, E_{02}\right]$. The critical curves separate the plane into two parts. When $\delta<\delta_{c}$, the clear states (or the turbid states) are nonlinearly stable; when $\delta>\delta_{c}$, the clear states (or the turbid states) are nonlinearly unstable and will have a regime shift. This also confirms the existence of the multiple equilibrium states for the shallow lake ecosystem. The results are shown in Figures 2(a) and 2(b).

Also, with the linear singular vector method, we get the LSVs. The obtained LSVs are different from the CNOP-Is under the same restraints. This also implies that the shallow lake ecosystem is governed by the nonlinear system. So, in this ecosystem, the nonlinear theory and the method should be considered. 


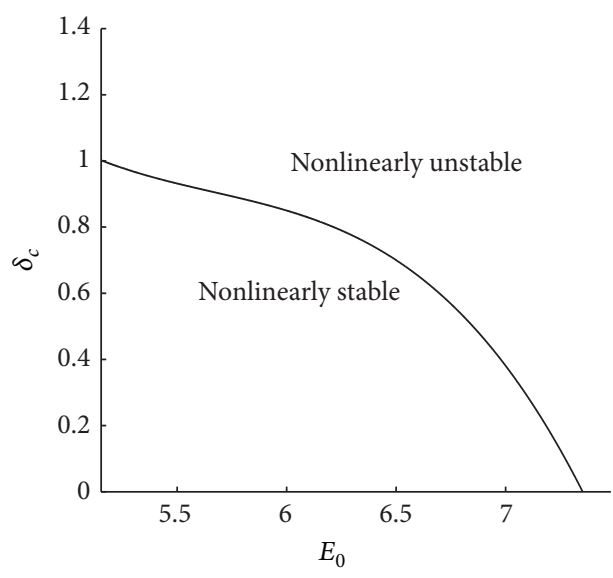

(a)

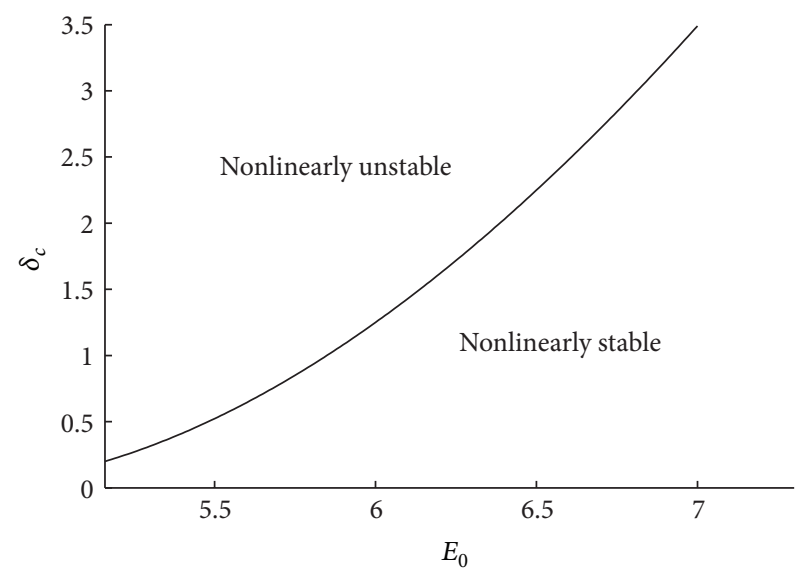

(b)

FIGURE 2: The critical value $\delta_{c}$ versus the control parameter $E_{0}$. (a) The basic states are CES; (b) the basic states are TES.

TABLE 1: CNOP-Ps corresponding to different ground states.

\begin{tabular}{cccccccc}
\hline$\sigma$ & $\begin{array}{c}\text { CNOP-Ps of CES A } \\
\left(E_{0}=4.0\right)\end{array}$ & $\sigma$ & $\begin{array}{c}\text { CNOP-Ps of TES B } \\
\left(E_{0}=8.0\right)\end{array}$ & $\sigma$ & $\begin{array}{c}\text { CNOP-Ps of CES C } \\
\left(E_{0}=6.0\right)\end{array}$ & $\begin{array}{c}\sigma \\
\text { CNOP-Ps of TES D } \\
\left(E_{0}=6.0\right)\end{array}$ \\
\hline 1.0 & -1.0 & 0.6 & -0.6 & 1.5 & -1.5 & 1.5 & -1.5 \\
3.0 & 3.0 & 3.9 & 3.9 & 5.0 & 5.0 & 4.0 & 4.0 \\
7.0 & 7.0 & 5.0 & 4.0 & 7.0 & 6.0 & 7.0 & 6.0 \\
\hline
\end{tabular}

3.2. The Impact of CNOP-Ps on the Shallow Lake Ecosystem. In realistic ecosystems, the perturbations which exist in some model parameters have a great impact on the shallow lakes. We only consider how the perturbations of the nutrients status affect the shallow lakes, so $E_{0}$ is just discussed in the parameters. Here, we still use SPG method to get the CNOPPs. In [6], we know that the control parameter $E_{0}$ belongs to the interval $[1.0,12.0]$, and so the parameter perturbation $E_{0}^{\prime}$ should satisfy both the condition $1.0 \leq E_{0}+E_{0}^{\prime} \leq 12.0$ and the restraint condition $\left|E_{0}^{\prime}\right| \leq \sigma$. Here, in the parameter interval $\left[1.0, E_{01}\right)$, we choose the control parameter $E_{0}=4.0$ and the corresponding clear stable equilibrium state CES A $V=$ $0.9872805862, E=0.6738087098$, with the constraints $\sigma=$ $1.0,3.0$, and 7.0 , respectively. In the interval $\left(E_{02}, 12.0\right]$, we choose the control parameter $E_{0}=8.0$ and the corresponding turbid stable equilibrium state TES B $V=0.0042293327$, $E=7.834330057$, with the constraints $\sigma=0.60,3.90$, and 5.0. In the interval $\left[E_{01}, E_{02}\right]$, we choose the control parameter $E_{0}=6.0$ and the corresponding clear stable equilibrium state CES C $V=0.925195745, E=1.066481104$, with the restraints $\sigma=1.50,5.0$, and 7.0, respectively; we choose the corresponding turbid stable state TES D $V=0.0167434949$, $E=5.536498341$, with the constraints $\sigma=1.5,4.0$, and 7.0. The obtained CNOP-Ps with the optimal time $\tau=100$, that is, 10 years, are shown in Table 1 . Also, we take the equilibrium state as the initial state and $E_{0}+C N O P-P$ as the new control parameter to integer the model with the integral time $T=$ 4000 , that is, 400 years. The results are shown in Figure 3.

Large amounts of numerical experiments show that there exists a critical restraint $\sigma_{c}$; when $\sigma<\sigma_{c}$, CNOP-P is negative and when $\sigma \geq \sigma_{c}$, CNOP-P is positive. Also, we can get the conclusion that when $E_{0}<E_{02}$ and the initial state is the stable CES, for $E_{0}+$ CNOP-P $\leq E_{02}$, the ecosystem will evolve from one stable CES to another stable CES; for $E_{02}<E_{0}+$ CNOP-P $\leq 12$, the ecosystem will evolve from the stable CES to the stable TES. When $E_{0}>E_{01}$ and the initial state is TES, if $E_{0}+$ CNOP-P $\geq E_{01}$, then the shallow lakes will evolve from the stable TES to another stable TES; if $1 \leq E_{0}+$ CNOP-P $<E_{01}$, the shallow lakes will transform from the stable TES to the stable CES.

3.3. The Impact of CNOPs on the Shallow Lake Ecosystem. Considering both the initial perturbations and the model parameter errors which satisfy the certain constraint conditions, respectively, we get the CNOPs which cause the largest departure from the reference states through the extended CNOP method. To calculate the CNOPs, we construct the adjoint model of the shallow lakes model (1) and obtain the gradient of the objective function with respect to the initial perturbations and the parameter perturbations that is similar to the computation of CNOP-Is and CNOP-Ps. With the obtained CNOPs superposed on the reference states, which are chosen to be the same to CNOP-Ps', we integer the dynamic model to get the nonlinear evolution of the ecosystem. Results are shown in Table 2 and Figure 4.

To facilitate the discussion, we hereafter use the coordinates $(\delta, \sigma)$ representing the amplitude of the initial perturbation and the parameter perturbation given while using the coordinates $\left(\mathrm{d} V, \mathrm{~d} E, \mathrm{~d} E_{0}\right)$ representing the CNOP obtained. Also, we use $E_{\text {new }}$ meaning the sum of $E_{0}$ and $\mathrm{d} E_{0}$ for short. A large amount of numerical experiments show that when $E_{\text {new }}<E_{01}$, no matter how large the $\mathrm{d} V$ and $\mathrm{d} E$ are, 


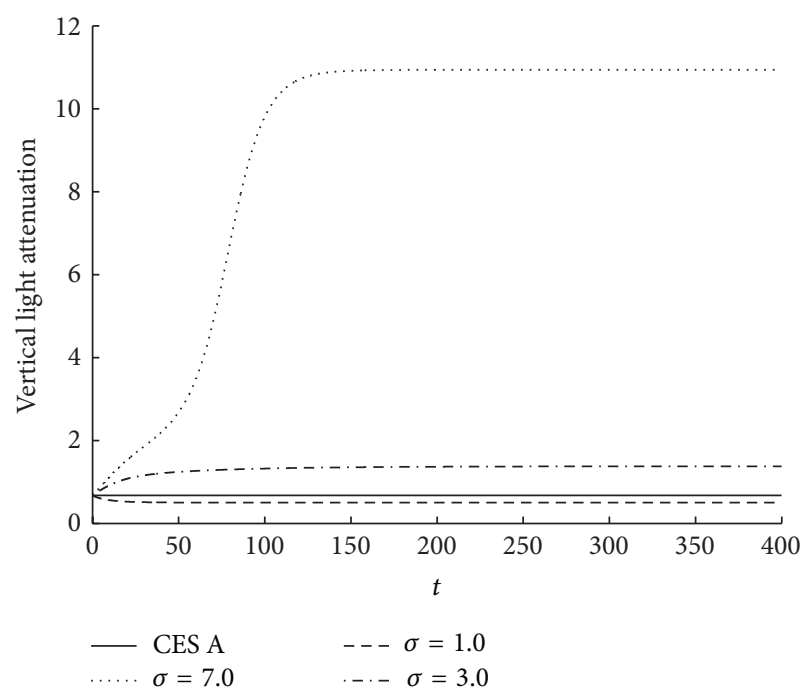

(a)

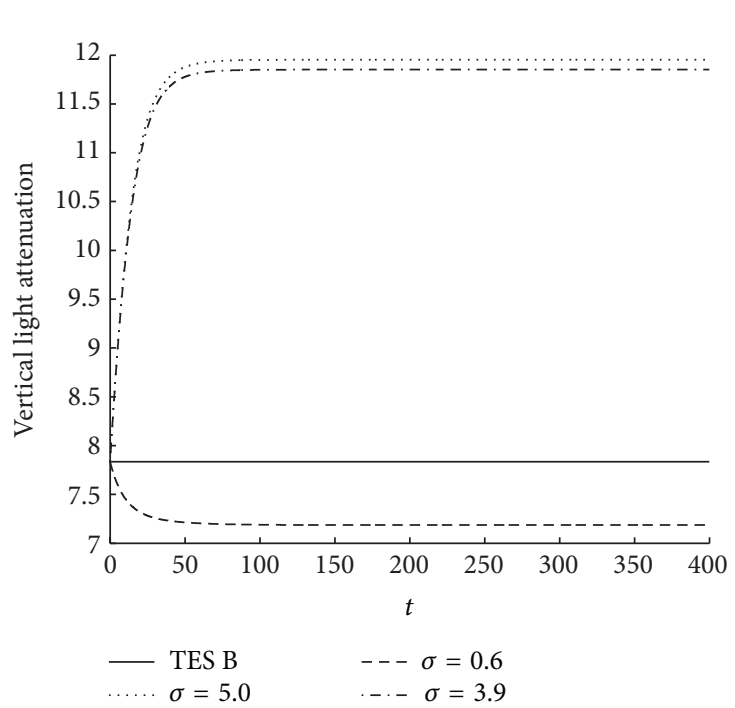

(c)

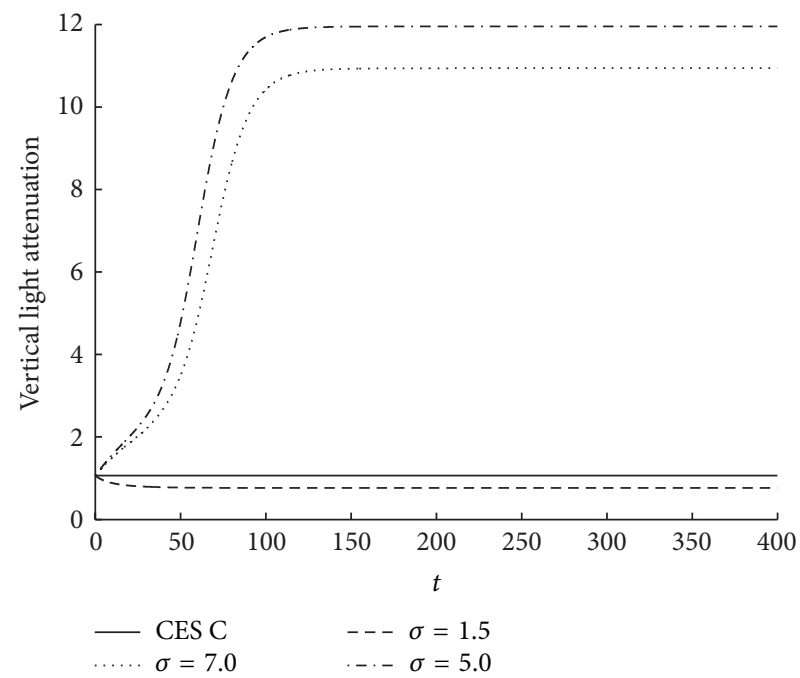

(e)

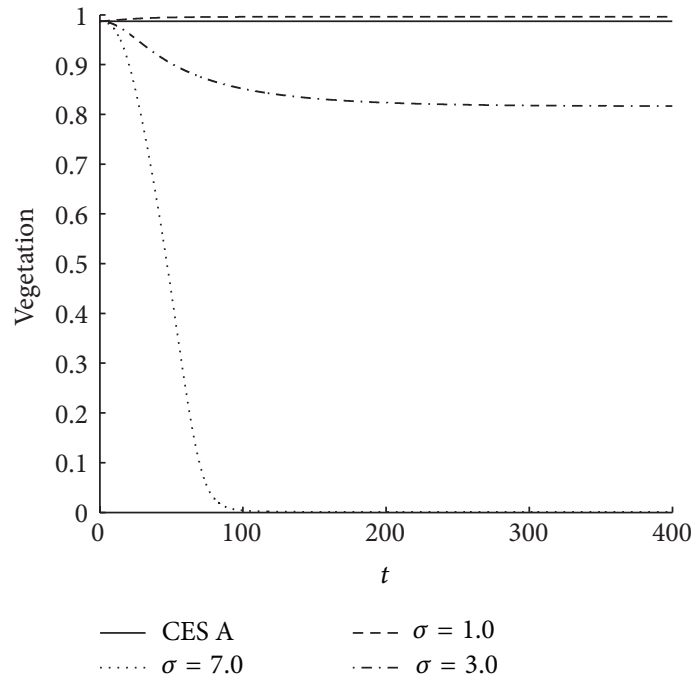

(b)

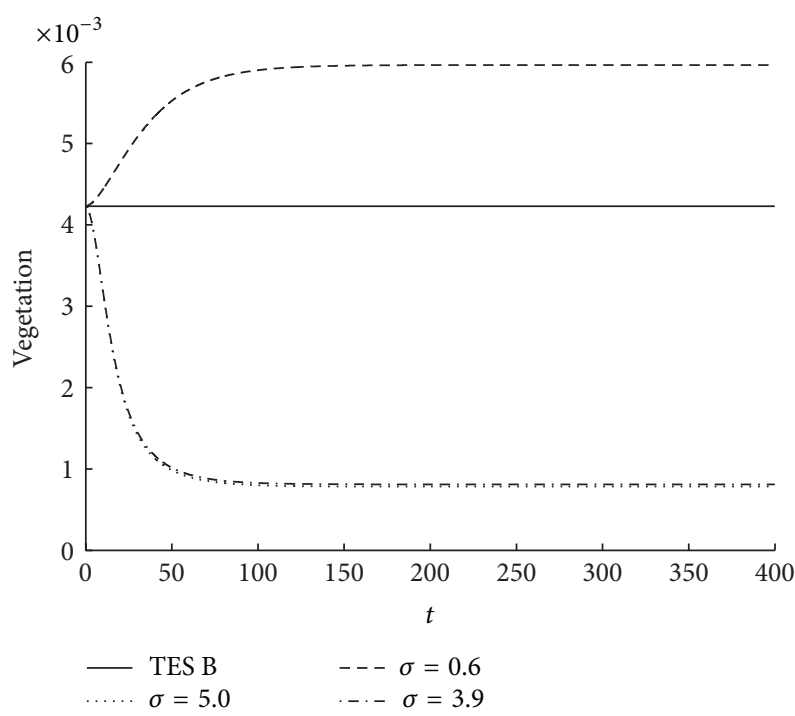

(d)

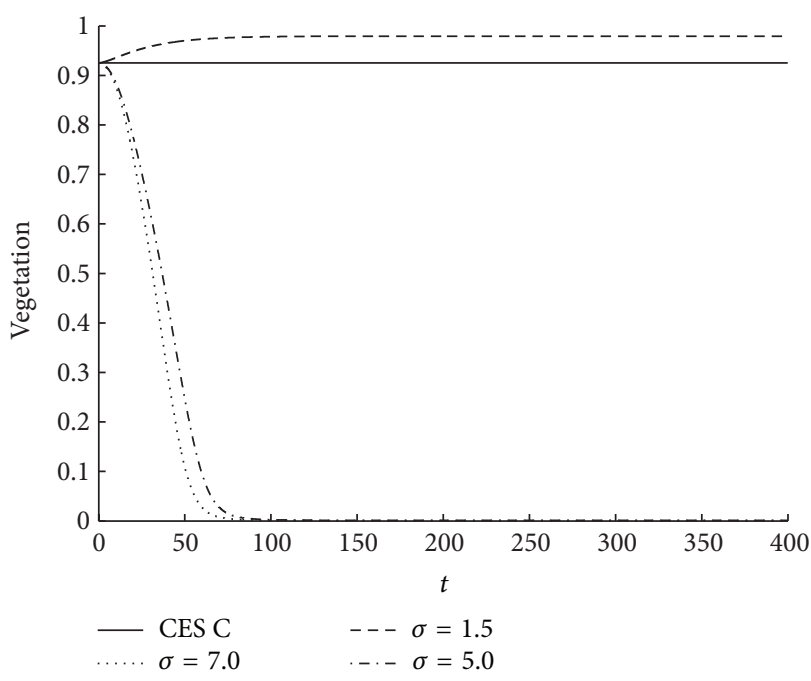

(f)

Figure 3: Continued. 


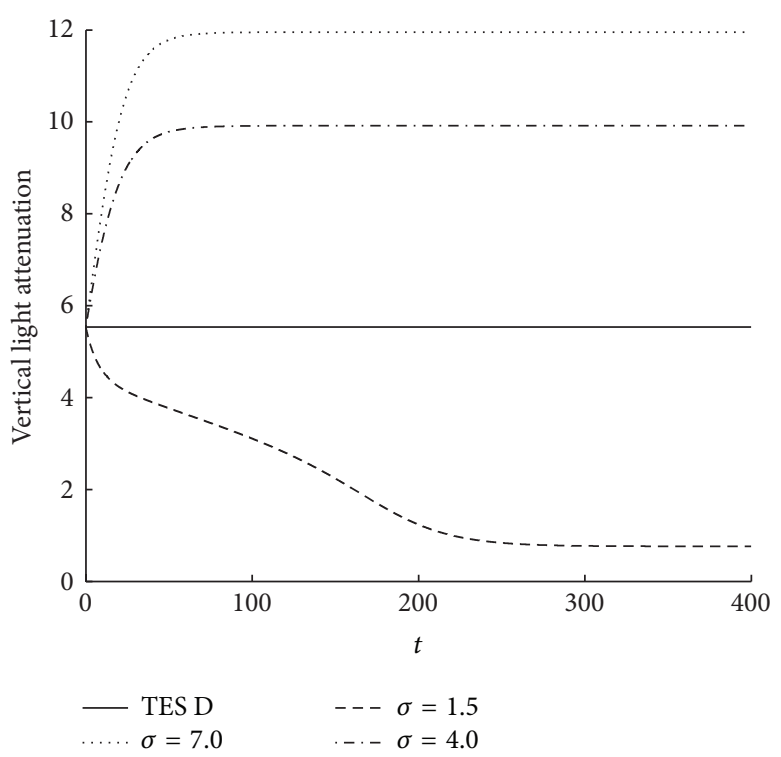

(g)

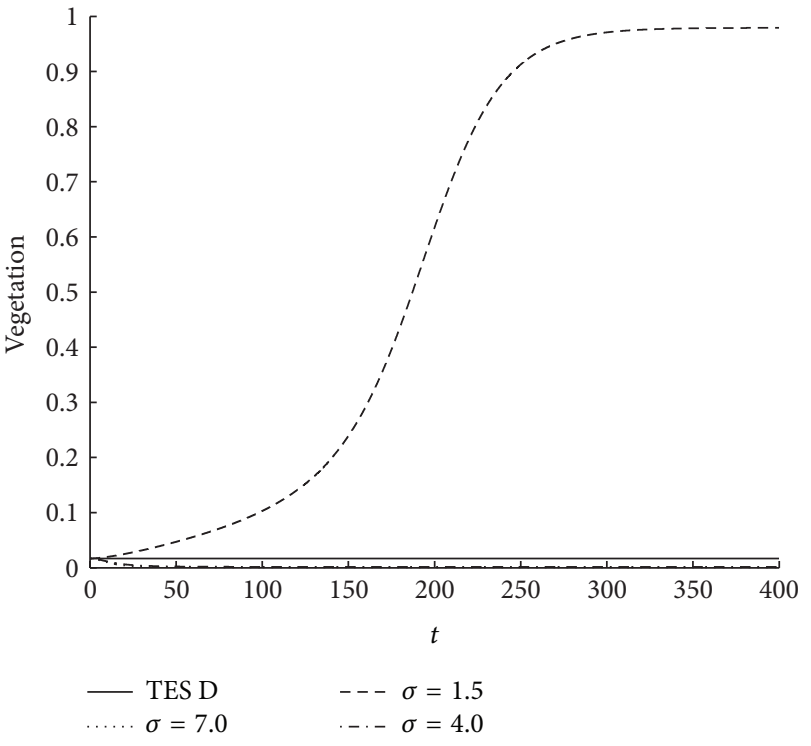

(h)

Figure 3: The nonlinear evolution of the shallow lake ecosystem, with the ground state as the initial condition and $E_{0}$ plus CNOP-P as the control parameter. (a) and (b) $E_{0}=4.0$, the ground state is CES A; (c) and (d) $E_{0}=8.0$, the ground state is TES B; (e) and (f) $E_{0}=6.0$, the ground state is CES C; $(\mathrm{g})$ and $(\mathrm{h}) E_{0}=6.0$, the ground state is TES D.

TABLE 2: CNOPs corresponding to different ground states.

\begin{tabular}{lccr}
\hline$(\delta, \sigma)$ & CNOPs of CES A $\left(E_{0}=4.0\right)$ & $(\delta, \sigma)$ & CNOPs of TES B $\left(E_{0}=8.0\right)$ \\
\hline$(0.5,2.0)$ & $(-0.43952238,0.23836963,2.0)$ & $(2.0,0.5)$ & $(0.58680515,-1.91197796,-0.5)$ \\
$(0.8,2.0)$ & $(-0.73880314,0.30687119,2.0)$ & $(1.5,1.0)$ & $(0.46880681,-1.42485795,-1.0)$ \\
$(0.8,1.0)$ & $(-0.75686935,0.25913085,1.0)$ & $(2.0,1.0)$ & $(0.61455195,-1.90324089,-1.0)$ \\
\hline$(\delta, \sigma)$ & CNOPs of CES C $\left(E_{0}=6.0\right)$ & $(\delta, \sigma)$ & CNOPs of TES D $\left(E_{0}=6.0\right)$ \\
\hline$(0.25,2.0)$ & $(-0.21108198,0.13395670,2.0)$ & $(0.45,1.0)$ & $(0.42240901,-0.15514713,-1.0)$ \\
$(0.45,2.0)$ & $(-0.40291733,0.20039368,2.0)$ & $(1.0,1.5)$ & $(0.81541862,-0.57887172,-1.5)$ \\
$(0.45,1.0)$ & $(-0.41347348,0.17759414,1.0)$ & &
\end{tabular}

the shallow lake ecosystem will turn to the CES corresponding to the control parameter $E_{\text {new }}$. When $E_{\text {new }}>E_{02}$, the ecosystem will turn to the TES corresponding to the control parameter $E_{\text {new }}$ no matter how large the $\mathrm{d} V$ and $\mathrm{d} E$ are. When $E_{01}<E_{\text {new }}<E_{02}$, if the reference state is CES corresponding to the control parameter $E_{0}$, then the ecosystem will turn to CES corresponding to the control parameter $E_{\text {new }}$ for the initial restraints $\delta<\delta_{c}$ and the ecosystem will turn to EES corresponding to the control parameter $E_{\text {new }}$ for the initial restraints $\delta>\delta_{c}$. If the reference state is TES corresponding to the control parameter $E_{0}$, then the ecosystem will turn to TES corresponding to the control parameter $E_{\text {new }}$ for the initial restraints $\delta<\delta_{c}$ and the ecosystem will turn to CES corresponding to the control parameter $E_{\text {new }}$ for the initial restraints $\delta>\delta_{c}$.

Now, we take the case of CES C and TES D corresponding to the control parameter $E_{0}=6$ as examples to explain the physical mechanism. For the initial state CES C, when the amplitude of the initial perturbation and the parameter perturbation satisfies $(\delta, \sigma)=(0.25,2.0)$ and $(\delta, \sigma)=(0.45,2.0)$, we can see that $E_{\text {new }}$ is 8.0 and $E_{\text {new }}>E_{02}$ from the CNOPs obtained, so the ecosystem will turn to the TES corresponding to the control parameter $E_{\text {new }}$. Figures $4(\mathrm{e})$ and $4(\mathrm{f})$ show that $\mathrm{d} E / \mathrm{d} t$ and $\mathrm{d} V / \mathrm{d} t$ are positive at about the first 15 years, so both the turbidity and the macrophytes increase. Then the turbidity continues increasing rapidly and the high turbidity changes the optical property in water. The lack of the light condition restrains the growing of the macrophytes and so the macrophytes begin to die gradually. At last, the lakes turn to the degenerated turbid states. Also, the figures show that the larger $\delta$ is, the less time it will take to turn to another stable state for the certain $\sigma$. When the amplitude of the initial perturbation and the parameter perturbation satisfies $(\delta, \sigma)=(0.45,1.0)$, we can see that $E_{\text {new }}$ is $7.0, \delta<$ $\delta_{c}$, and $E_{01}<E_{\text {new }}<E_{02}$ from the CNOPs obtained. So the ecosystem will turn to CES corresponding to the control parameter $E_{\text {new }}$ by the analysis above. And this also can be tested by Figures $4(\mathrm{e})$ and $4(\mathrm{f})$. For the $(\delta, \sigma)=$ $(0.45,1.0)$, we can see that $\mathrm{d} E / \mathrm{d} t$ and $\mathrm{d} V / \mathrm{d} t$ are positive, and both the turbidity and the macrophytes increase. Then, the turbidity stops increasing and the low turbidity in water will provide enough light conditions, so the macrophytes begin 


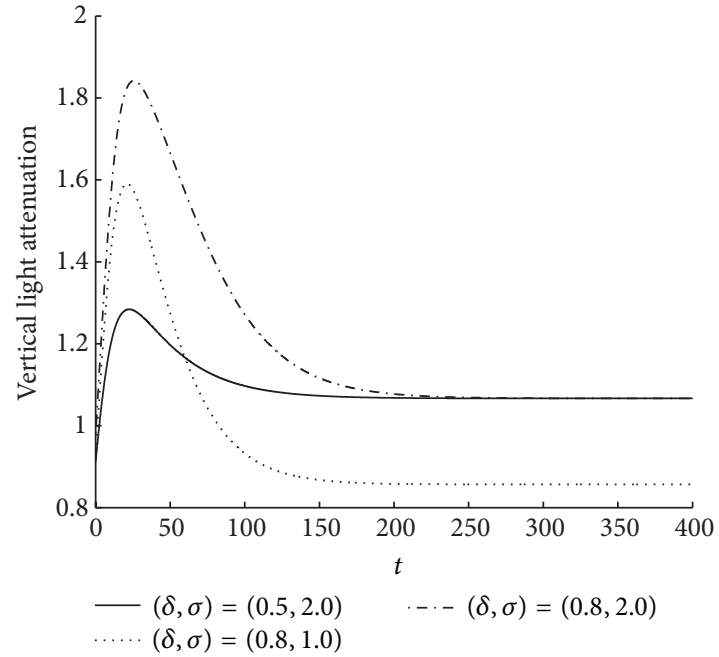

(a)

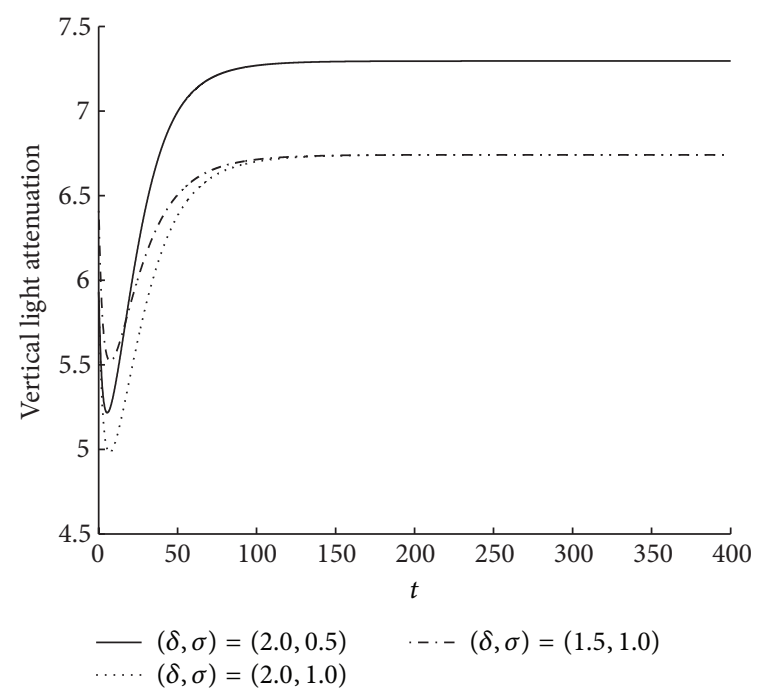

(c)

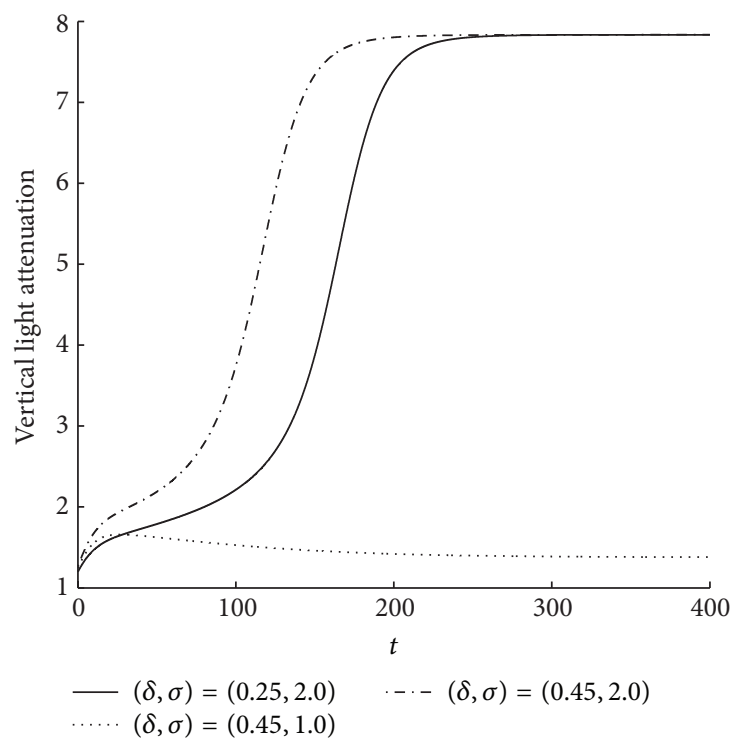

(e)

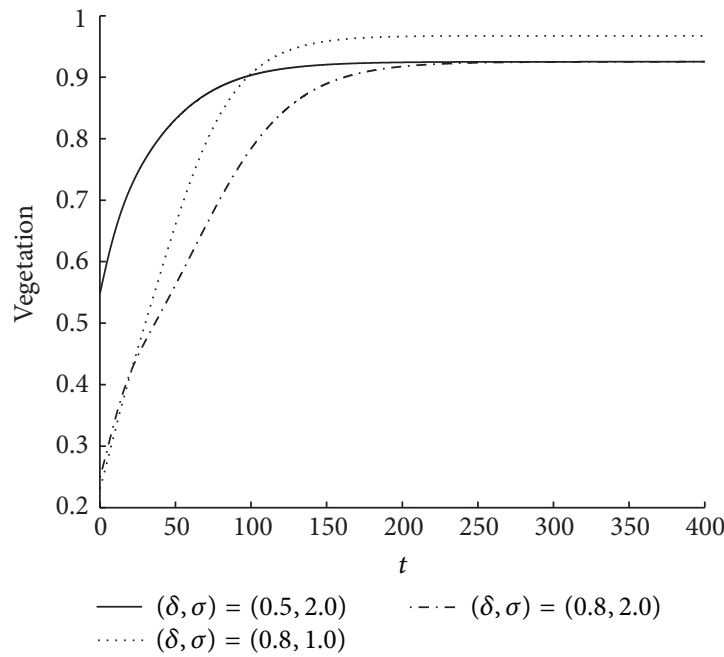

(b)

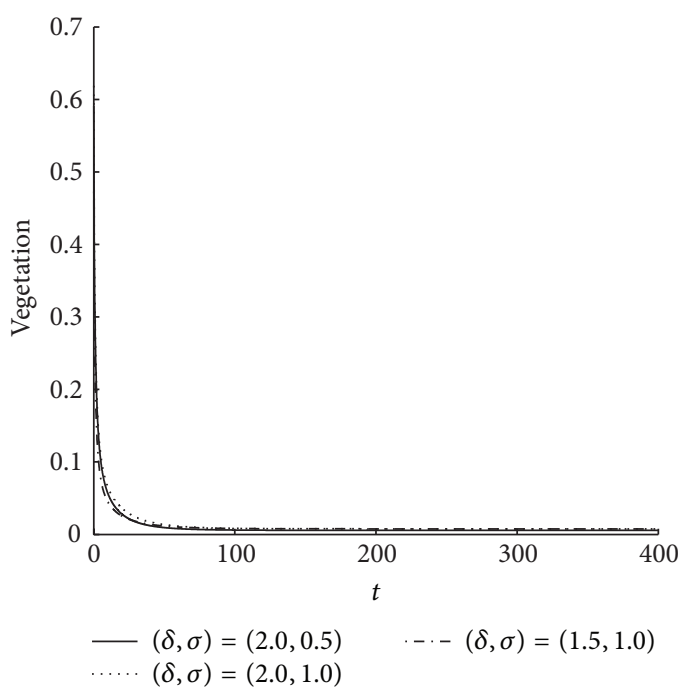

(d)

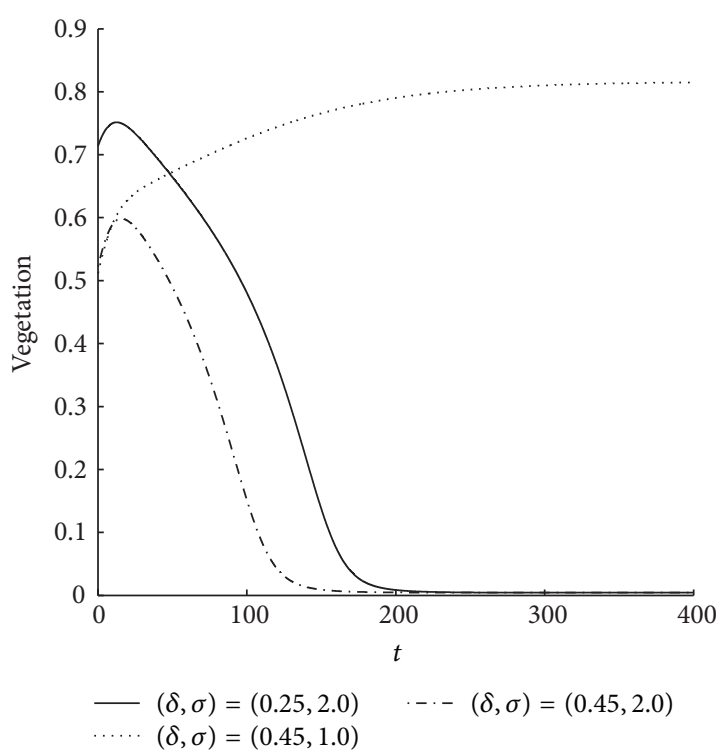

(f)

FIgURE 4: Continued. 


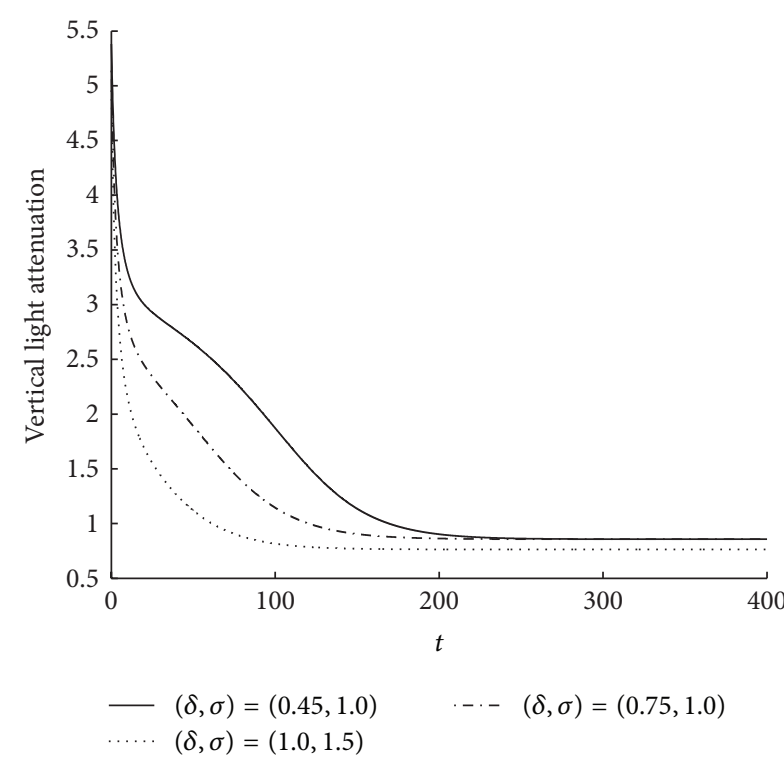

(g)

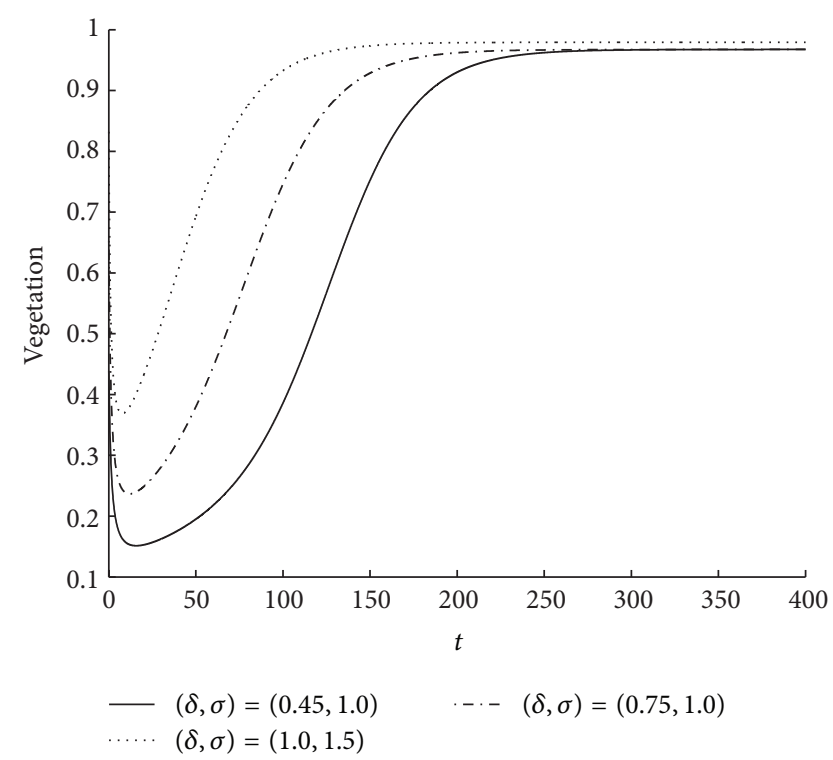

(h)

FIGURE 4: The nonlinear evolution of the shallow lake ecosystem, with original initial state plus $\mathrm{d} V$ and $\mathrm{d} E$ in CNOP as the new initial state and $E_{0}$ plus $\mathrm{d} E_{0}$ in CNOP as the control parameter. (a) and (b) $E_{0}=4.0$, the ground state is CES A; (c) and (d) $E_{0}=8.0$, the ground state is TES B; (e) and (f) $E_{0}=6.0$, the ground state is CES C; $(\mathrm{g})$ and $(\mathrm{h}) E_{0}=6.0$, the ground state is TES D.

to grow gradually. Macrophytes can reduce the sediment resuspension and absorb the nutrients from the water and this will increase the water transparency. The feedback between macrophytes and the turbidity makes the ecosystem evolve to our expected stable states.

For the initial state TES D, when the amplitude of the initial perturbation and the parameter perturbation satisfies $(\delta, \sigma)=(0.45,1.0)$ and $(\delta, \sigma)=(0.75,1.0)$, we can see that $E_{\text {new }}$ is 5.0 and $E_{\text {new }}<E_{01}$ with the CNOPs we have obtained. Also, for $(\delta, \sigma)=(1.0,1.5)$, from the CNOPs obtained, we can see that $E_{\text {new }}$ is 4.5 and still $E_{\text {new }}<E_{01}$. So the ecosystem will turn to the CES corresponding to the control parameter $E_{\text {new. }}$. Figures $4(\mathrm{~g})$ and $4(\mathrm{~h})$ show that $\mathrm{d} E / \mathrm{d} t$ and $\mathrm{d} V / \mathrm{d} t$ are negative at about the first 10 years, so both the turbidity and the macrophytes decrease rapidly. Then the turbidity continues decreasing to the lower state, so the high transparency in water provides the proper growing condition for the macrophytes. Then the macrophytes begin to grow rapidly. Further, the macrophytes absorb the nutrient in water and restrain the resuspension in sediment. At last, the shallow lakes will keep the high transparency and macrophyte covers and the ecosystem will transfer to the clear water stable states. For other control parameters $E_{0}$ and other restraints at different states, we have the similar conclusion and the physical mechanism. Here, details are omitted.

\section{The Comparison with Different Optimal Perturbation for the Ecosystem}

If only considering the initial perturbations, CNOP-Is induced the largest departure from the reference state; if only considering the model parameter's perturbations, CNOPPs have the greatest impact on the shallow lakes; when considering both the initial perturbations and the parameter perturbations, CNOPs have the largest effect on the ecosystem. Then it is required to know which combined mode of the initial perturbation and parameter perturbation yields the maximum effect on the shallow lake ecosystem. Also, it is desired to know that whether the simple combination of CNOP-I and CNOP-P is equal to CNOP. For simplicity, we call the simple combination of CNOP-I and CNOP-P (CNOP-I, CNOP-P) for short.

Here, we take CES A in the interval $\left[1.0, E_{01}\right]$, TES B in the interval $\left[E_{02}, 12.0\right]$, and CES C, TES D in the interval $\left[E_{01}, E_{02}\right]$ as examples to illustrate the conclusion by comparing the objective function which is defined in (5). To show the result clearly, we give the CNOP-Is, CNOPPs, and CNOPs, (CNOP-I, CNOP-P) and the corresponding objective function values related to the equilibrium states with different restraint in Table 3. Also, we make the column diagram in Figure 5.

The above results show that CNOPs could affect the shallow lakes at the most extent. In addition, CNOP is not the simple combination of CNOP-I and CNOP-P. From the results, we can also see that (CNOP-I, CNOP-P) may decrease the impact of CNOP-I or CNOP-P on the ecosystem sometimes.

In addition, to discuss the sensitivities of the shallow lake ecosystem to the (CNOP-I, CNOP-P), for simplicity, $E_{0}+$ CNOP-P is denoted by $E_{\text {new } 1}$ and $\delta_{c}$ means the critical initial restraint corresponding to control parameter $E_{\text {newl }}$. Plenty of numerical results imply that when $E_{\text {newl }}<E_{01}$, no matter how large the CNOP-I is, the shallow lake ecosystem will turn to the CES corresponding to the control parameter 
TABLE 3: CNOP-I, CNOP-P, CNOP, and (CNOP-I, CNOP-P) corresponding to different ground states and the corresponding objective function values, where obj means the objective function values.

\begin{tabular}{lcccccc}
\hline Basic state & $\delta$ & $\sigma$ & CNOP-I & CNOP-P & CNOP & $($ CNOP-I, CNOP-P) \\
\hline CES A & 0.8 & 1.0 & $(-0.772944,0.206295)$ & -1.0 & $(-0.756869,0.259131,1.0)$ & $(-0.772944,0.206295,-1.0)$ \\
obj & & & 0.89778821 & 0.11352425 & 0.99494645 & 0.79613468 \\
TES B & 1.5 & 1.0 & $(0.424979,-1.438538)$ & -1.0 & $(0.468807,-1.424858,-1.0)$ & $(0.424979,-1.438538,-1.0)$ \\
obj & & & 1.65155171 & 0.63125732 & 2.28696025 & 2.28461456 \\
CES C & 0.45 & 1.0 & $(-0.426888,0.142361)$ & -1.0 & $(-0.413473,0.177594,1.0)$ & $(-0.426888,0.142361,-1.0)$ \\
obj & & & 0.48494774 & 0.11790117 & 0.58937605 & 0.38767470 \\
TES D & 1.5 & 2.0 & $(0.956400,-1.155551)$ & 2.0 & $(0.981794,-1.134055,-2.0)$ & $(0.956400,-1.155551,2.0)$ \\
obj & & & 3.11289007 & 1.03696634 & 4.11767312 & 2.07934424 \\
\hline
\end{tabular}

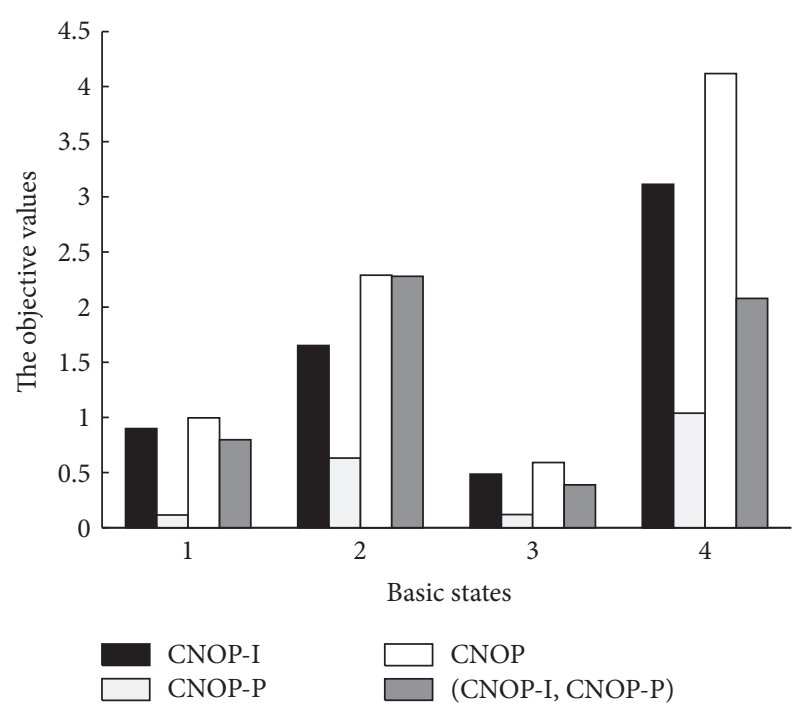

FIgUre 5: The objective values caused by the CNOP-I, CNOP-P, and CNOP and the combination of CNOP-I and CNOP-P. The numbers 1,2,3, and 4 in the horizontal axis denote the four different basic states, respectively. 1 represents that the basic state is CES A; 2 represents that the basic state is TES B; 3 represents that the basic state is CES C; 4 represents that the basic state is TES D. The measurement of the objective function values is $J\left(u_{0} ; p^{\prime}\right)=$ $\sqrt{(V(\tau)-V(0))^{2}+(E(\tau)-E(0))^{2}}$.

$E_{\text {newl }}$. When $E_{\text {newl }}>E_{02}$, the ecosystem will turn to the TES corresponding to the control parameter $E_{\text {newl }}$ no matter how large the CNOP-I is. When $E_{01}<E_{\text {new1 }}<E_{02}$, if the basic state is CES corresponding to the control parameter $E_{0}$, then the ecosystem will turn to CES corresponding to the control parameter $E_{\text {newl }}$ for the initial restraints $\delta<\delta_{c}$ and the ecosystem will turn to EES corresponding to the control parameter $E_{\text {new } 1}$ for the initial restraints $\delta>\delta_{c}$. If the basic state is TES corresponding to the control parameter $E_{0}$, then the ecosystem will turn to TES corresponding to the control parameter $E_{\text {newl }}$ for the initial restraint $\delta<\delta_{c}$ and the ecosystem will turn to CES corresponding to the control parameter $E_{\text {newl }}$ for the initial restraints $\delta>\delta_{c}$.

Compared with CNOP, the results show that when we choose the same control parameter $E_{0}$, the same basic state, and furthermore the same initial perturbations and parameter perturbations restraint, if $E_{\text {newl }}$ is equal to $E_{\text {new }}$, CNOP and (CNOP-I, CNOP-P) induce the shallow lake ecosystem into the same finial state at the end of the integral time $T$; when $E_{\text {newl }}$ is not equal to $E_{\text {new }}, C N O P$ and (CNOP-I, CNOP-P) induce the shallow lake ecosystem into the different finial state at $T$, while it depends on the $E_{\text {new }}$ and $E_{\text {new1 }}$.

Large numbers of experiments also show that when the value of the third component in CNOP is equal to the value of the CNOP-P in (CNOP-I, CNOP-P) under the same restraint, the objective values which are caused by CNOP are closed to those ones which are caused by (CNOP-I, CNOP-P) at the end of the optimal time $\tau$; that is to say, CNOP could induce the shallow lake ecosystem slightly larger departure from the basic state than (CNOP-I, CNOP-P) at $\tau$; when the value of the third component in CNOP is not equal to the value of the CNOP-P in (CNOP-I, CNOP-P) under the same restraint, the objective values which are caused by CNOP and (CNOP-I, CNOP-P), respectively, vary widely; that is to say, CNOP could induce the shallow lake ecosystem much larger departure from the reference state at time $\tau$ than (CNOP-I, CNOP-P).

\section{Conclusions}

With a two-variable shallow lake model, we have discussed the nonlinear stability and sensitivity of the ecosystem to the finite-amplitude initial perturbation and the parameter perturbation. The results obtained show that the shallow lake ecosystem may transfer to another equilibrium state with a large enough CNOP-I as the initial perturbation, a large enough CNOP-P as the parameter perturbation, or a large enough CNOP, both including the initial perturbation and the parameter perturbation. From the above analysis, we can also verify that the positive feedback between the macrophytes and the transparency in water is the main mechanism which triggers the ecosystem to have a regime shift.

In addition, $\mathrm{CNOP}$ affects the ecosystem most compared to CNOP-I, CNOP-P, and (CNOP-I, CNOP-P) with the same initial perturbation constraint $\delta$ and the same parameter perturbation constraint $\sigma$. CNOP is not the simple combination of CNOP-I and CNOP-P, which may affect the ecosystem less than both CNOP-I and CNOP-P.

In our future work, we will try to explore how the optimal model of multiple parameter perturbation affects the shallow 
lake ecosystem and which types and which patterns of the parameter perturbations could lead to the most unstable modeled shallow lake ecosystem.

\section{Conflict of Interests}

The authors declare that there is no conflict of interests regarding the publication of this paper.

\section{Acknowledgment}

This research was supported by the National Natural Science Foundation of China (Grant no. 40805020).

\section{References}

[1] M. Scheffer, S. Rinaldi, A. Gragnani, L. R. Mur, and E. H. Van Nes, "On the dominance of filamentous cyanobacteria in shallow, turbid lakes," Ecology, vol. 78, no. 1, pp. 272-282, 1997.

[2] M. Scheffer, S. Szabó, A. Gragnani et al., "Floating plant dominance as a stable state," Proceedings of the National Academy of Sciences of the United States of America, vol. 100, no. 7, pp. 40404045, 2003.

[3] B. E. Beisner, D. T. Haydon, and K. Cuddington, "Alternative stable states in ecology," Frontiers in Ecology and the Environment, vol. 1, no. 7, pp. 376-382, 2003.

[4] S. Harrison, "Ecology of shallow lakes by M. Scheffer-a book review," Journal of Ecology, vol. 86, no. 6, pp. 1075-1076, 1998.

[5] M. Scheffer and S. R. Carpenter, "Catastrophic regime shifts in ecosystems: linking theory to observation," Trends in Ecology and Evolution, vol. 18, no. 12, pp. 648-656, 2003.

[6] E. H. Van Nes and M. Scheffer, "Implications of spatial heterogeneity for catastrophic regime shifts in ecosystems," Ecology, vol. 86, no. 7, pp. 1797-1807, 2005.

[7] G. J. Van Geest, H. Coops, M. Scheffer, and E. H. Van Nes, "Long transients near the ghost of a stable state in eutrophic shallow lakes with fluctuating water levels," Ecosystems, vol. 10, no. 1, pp. 36-46, 2007.

[8] M. Scheffer, Ecology of Shallow Lakes, Chapman and Hall, New York, NY, USA, 1998.

[9] M. Mu, W. S. Duan, and B. Wang, "Conditional nonlinear optimal perturbation and its applications," Nonlinear Processes in Geophysics, vol. 10, no. 6, pp. 493-501, 2003.

[10] M. Mu, W. Duan, Q. Wang, and R. Zhang, "An extension of conditional nonlinear optimal perturbation approach and its applications," Nonlinear Processes in Geophysics, vol. 17, no. 2, pp. 211-220, 2010.

[11] G. D. Sun, M. Mu, and Y. L. Zhang, "Algorithm studies on how to obtain a conditional nonlinear optimal perturbation (CNOP)," Atmospheric Sciences, vol. 27, no. 6, pp. 1311-1321, 2010.

[12] M. Mu and W. S. Duan, "A new approach to studying ENSO predictability: conditional nonlinear optimal perturbation," Chinese Science Bulletin, vol. 48, no. 10, pp. 1045-1047, 2003.

[13] W. S. Duan, M. Mu, and B. Wang, "Conditional nonlinear optimal perturbations as the optimal precursors for El NinoSouthern Oscillation events," Journal of Geophysical Research D, vol. 109, no. 23, 12 pages, 2004.

[14] W. S. Duan and M. Mu, "Investigating decadal variability of El Nino-Southern Oscillation asymmetry by conditional nonlinear optimal perturbation," Journal of Geophysical Research C, vol. 111, no. 7, Article ID C07015, 2006.
[15] W. S. Duan, F. Xue, and M. Mu, "Investigating a nonlinear characteristic of El Niño events by conditional nonlinear optimal perturbation," Atmospheric Research, vol. 94, no. 1, pp. 10-18, 2009.

[16] W. S. Duan, H. Xu, and M. Mu, "Decisive role of nonlinear temperature advection in El Niño and La Niña amplitude asymmetry," Journal of Geophysical Research C, vol. 113, no. 1, Article ID C01014, 2008.

[17] W. S. Duan and M. Mu, "Conditional nonlinear optimal perturbation: applications to stability, sensitivity, and predictability," Science in China, Series D: Earth Sciences, vol. 52, no. 7, pp. 883906, 2009.

[18] W. S. Duan, X. C. Liu, K. Y. Zhu, and M. Mu, "Exploring the initial errors that cause a significant "spring predictability barrier" for El Niño events," Journal of Geophysical Research C: Oceans, vol. 114, no. 4, Article ID C04022, 2009.

[19] W. S. Duan and H. Y. Luo, "A new strategy for solving a class of constrained nonlinear optimization problems related to weather and climate predictability," Advances in Atmospheric Sciences, vol. 27, no. 4, pp. 741-749, 2010.

[20] W. S. Duan and R. Zhang, "Is model parameter error related to a significant spring predictability barrier for El Niño events? Results from a theoretical model," Advances in Atmospheric Sciences, vol. 27, no. 5, pp. 1003-1013, 2010.

[21] M. Mu and B. Wang, "Nonlinear instability and sensitivity of a theoretical grassland ecosystem to finite-amplitude perturbations," Nonlinear Processes in Geophysics, vol. 14, no. 4, pp. 409423, 2007.

[22] B. Wang and Z. Huo, "Extended application of the conditional nonlinear optimal parameter perturbation method in the Common Land Model," Advances in Atmospheric Sciences, vol. 30, no. 4, pp. 1213-1223, 2013.

[23] B. Wang, Z. H. Huo, Q. Q. Qi, and P. J. Zhang, "The research on the lake eutrophication with CNOP method," Advanced Materials Research, vol. 599, pp. 705-708, 2012.

[24] Q. Q. Qi and B. Wang, "Application of the CNOP method in the shallow lake ecosystem," Advanced Materials Research, vol. 1010-1012, pp. 658-661, 2014. 

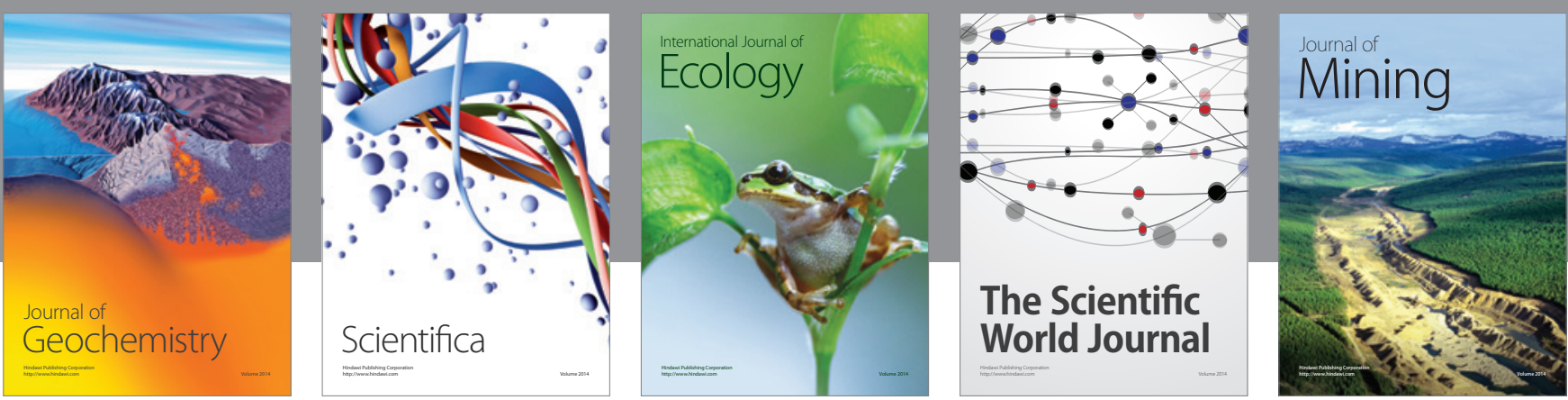

The Scientific World Journal
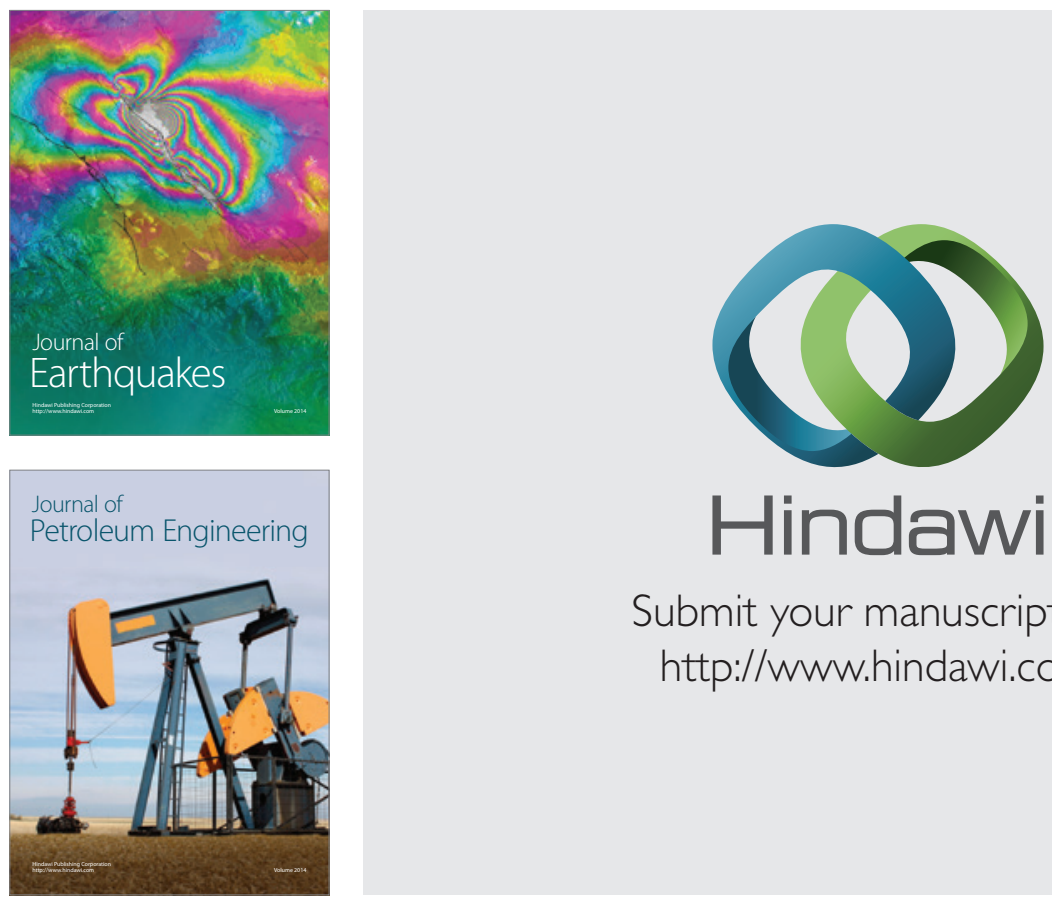

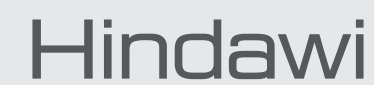

Submit your manuscripts at

http://www.hindawi.com
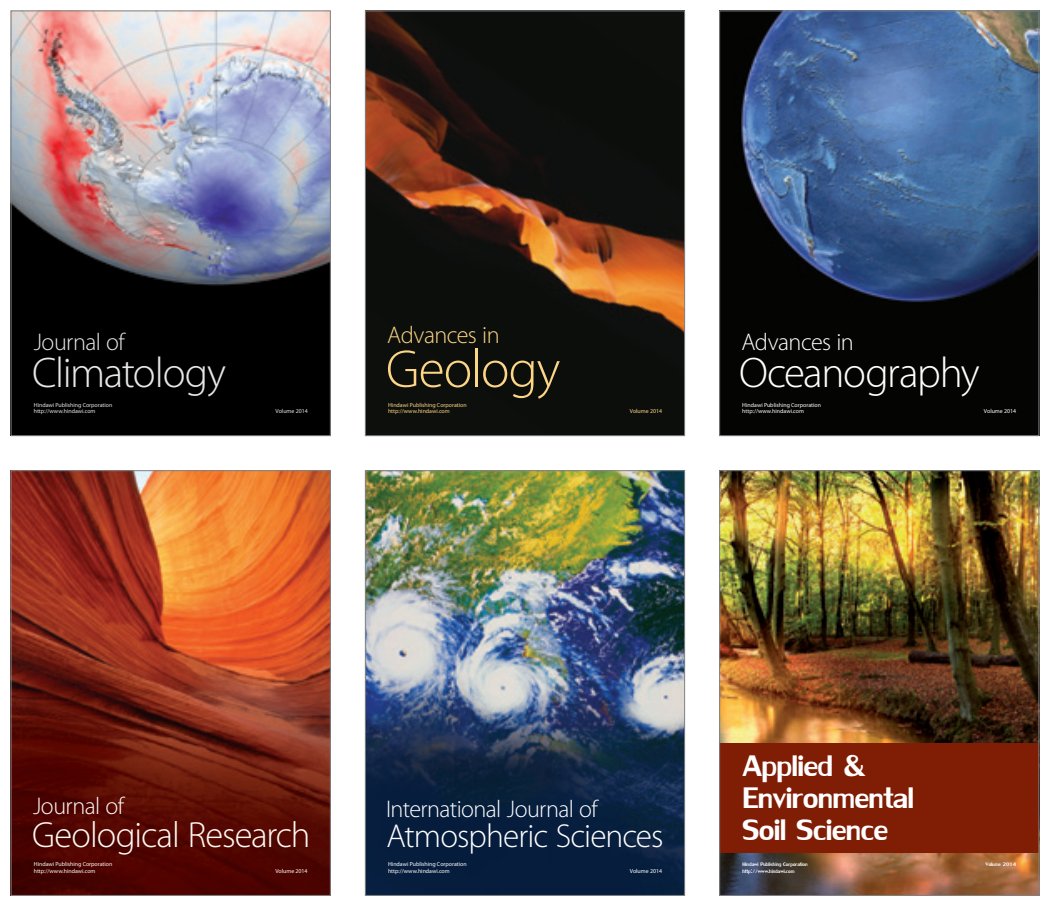
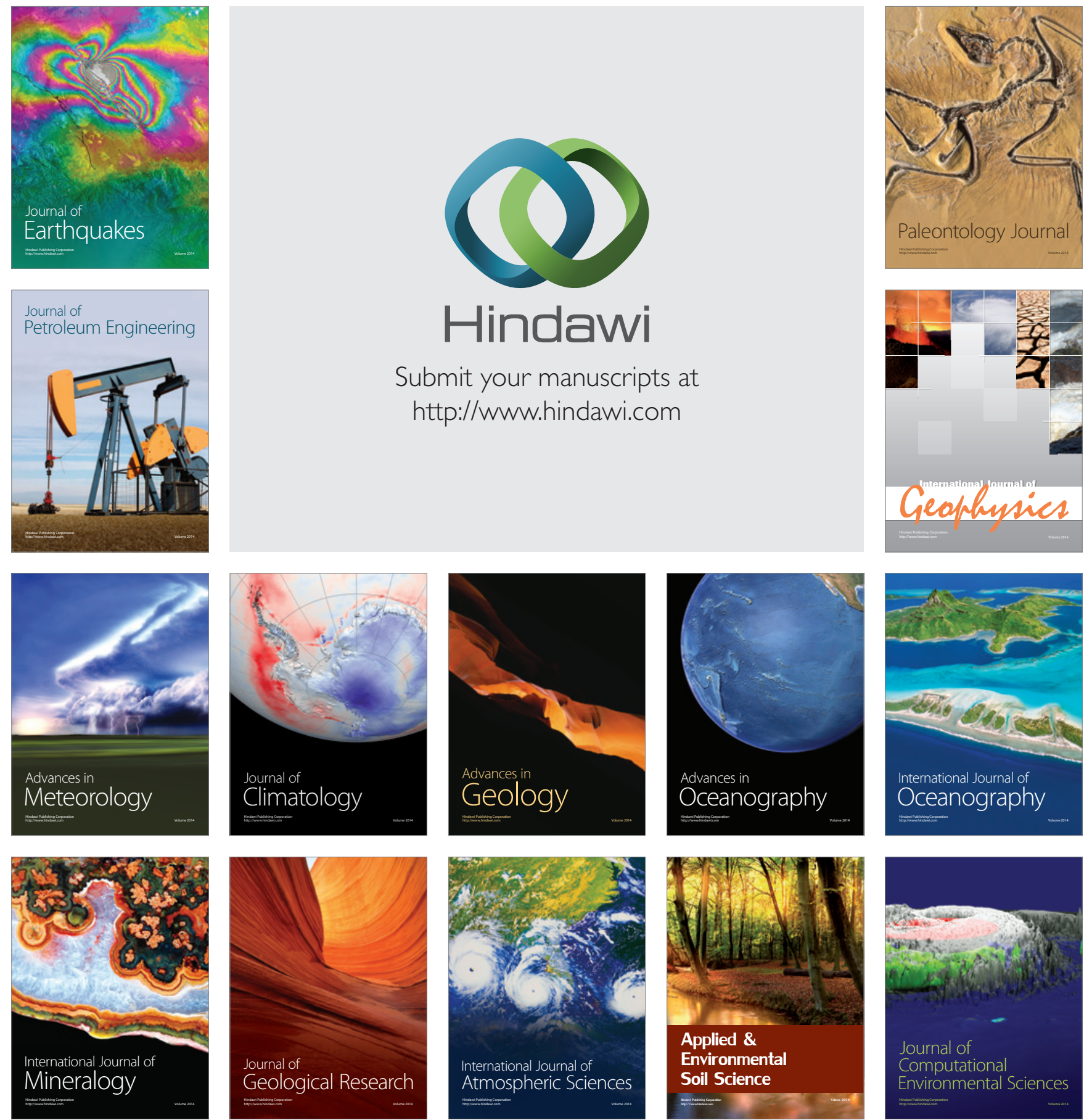